\title{
Multiwavelength study of the very long GRB 020410
}

\author{
L. Nicastro ${ }^{1}$, J. J. M. in 't Zand ${ }^{2,3}$, L. Amati ${ }^{6}$, S. Golenetskii ${ }^{4}$, A. Castro-Tirado ${ }^{5}$, J. Gorosabel ${ }^{5}$, D. Lazzati ${ }^{7}$, \\ E. Costa ${ }^{8}$, M. De Pasquale ${ }^{8}$, M. Feroci ${ }^{8}$, J. Heise ${ }^{2,3}$, E. Pian ${ }^{9}$, L. Piro ${ }^{8}$, C. Sánchez-Fernández ${ }^{10}$, and P. Tristram ${ }^{11}$ \\ ${ }^{1}$ IASF - CNR, via U. La Malfa 153, 90146 Palermo, Italy \\ e-mail: nicastro@pa.iasf.cnr.it \\ 2 SRON National Institute for Space Research, Sorbonnelaan 2, 3584 CA Utrecht, The Netherlands \\ 3 Astronomical Institute, Utrecht University, PO Box 80000, 3508 TA Utrecht, The Netherlands \\ ${ }^{4}$ Ioffe Institute, 26 Polytekhnicheskaya, St. Petersburg 194021, Russian Federation \\ 5 Instituto de Astrofísica de Andalucía (IAA-CSIC), PO Box 03004, 18080 Granada, Spain \\ ${ }^{6}$ IASF - CNR, via P. Gobetti 101, 40129 Bologna, Italy \\ 7 Institute of Astronomy, University of Cambridge, Madingley Road, UK CB3 OHA Cambridge, UK \\ 8 IASF - CNR, via Fosso del Cavaliere, 00131 Roma, Italy \\ 9 Osservatorio Astr. di Trieste, via GB Tiepolo 11, 34131 Trieste, Italy \\ 10 XMM-Newton Science Operations Centre, Villafranca del Castillo, PO Box 50727, 28080 Madrid, Spain \\ 11 Mt. John University Observatory, Canterbury University, New Zealand
}

Received 24 March 2004 / Accepted 16 July 2004

\begin{abstract}
GRB 020410 is by far the longest $\gamma$-ray burst (with a duration of about $1600 \mathrm{~s}$ ) to have been followed up from the $\mathrm{X}$-ray through the radio regime. Afterglow emission was detected in X-rays and at optical wavelengths whereas no emission was detected at $8 \mathrm{GHz}$ brighter than $120 \mu \mathrm{Jy}$. The decaying X-ray afterglow, back-extrapolated to $11 \mathrm{~h}$ after the burst, had a flux of $7.9 \times 10^{-12} \mathrm{erg} \mathrm{cm}^{-2} \mathrm{~s}^{-1}(2-10 \mathrm{keV})$; the brightest detected so far. No direct redshift determination is available yet for this GRB, but according to the empirical relationship between the peak energy in the $v F_{v}$ spectrum and the isotropic energy output, $z$ is constrained in the range 0.9-1.5. The reconstructed optical afterglow light curve implies at least two breaks in the simple power law decay. This may be related to emergence of an $\mathrm{SN}$, or refreshment of the external shock by a variation in the circumstellar medium. Considering the backward extrapolation of the $2-10 \mathrm{keV}$ afterglow decay, the prompt lightcurve variability and its spectral evolution, we conclude that the long duration of this event is due to a prolonged activity of the "central engine".
\end{abstract}

Key words. gamma rays: bursts - X-rays: bursts

\section{Introduction}

Gamma-ray Bursts (GRB) show great diversity with regard to both their durations and spectral properties. GRBs last from a fraction of a second to thousands of seconds, as established by the BATSE survey (e.g. Paciesas et al. 1999). Prompt X-ray counterparts of GRBs, detected by Ginga, BeppoSAX, HETE-2 have a very wide distribution of intensities and durations. Tails and precursors of X-ray counterparts were also observed by WATCH/Granat (Castro-Tirado et al. 1994). Those events characterized by an X-rays-to- $\gamma$-rays $(2-10 / 40-700 \mathrm{kev})$ fluence ratio larger than $\sim 0.5$ are classified as X-ray rich (e.g. Feroci et al. 2001). Moreover, transient $X$-ray sources with characteristics similar to those of GRB counterparts, although with no simultaneous GRB detection (so called "X-ray flashes"; Heise et al. 2001; Mochkovitch et al. 2003) were detected by the BeppoSAX Wide Field Cameras (WFC) and, subsequently, by the HETE-2/FREGATE instrument. Recently in 't Zand et al. (2003) have reported the detection of 4 long, faint X-ray transients during sky surveys with the BeppoSAX-WFC. Three of these are confirmed GRBs, because they coincide with BATSE detections. They have durations ranging from $540 \mathrm{~s}$ to $2550 \mathrm{~s}$ and are characterized by a mildly soft spectrum.

The very different ratios of $\gamma$-ray vs. X-ray peak fluxes or fluences point either to different viewing angles of the relativistic jets in which GRBs are formed (e.g. Granot et al. 2002; Yamazaki et al. 2002) or to a different amount of baryon contamination of the fireball (e.g. Dermer 1999; Huang et al. 2002). Furthermore, the existence of a class of GRBs with long $\mathrm{X}$-ray durations is important for the investigation of the connection between the prompt and afterglow components and may also suggest a high redshift origin. However, redshift constraints imposed on XRF 020903, 030723 and, possibly, 031203 do not support the high redshift scenario (Soderberg et al. 2003; Prochaska et al. 2003; Fynbo et al. 2004).

GRB 020410, first detected in X-rays only by the BeppoSAX-WFC (Gandolfi 2002), stands out for its long duration, more than $1500 \mathrm{~s}$ in the $2-28 \mathrm{keV}$ band (see Sect. 3.1), 
and for the relative weakness of its $\gamma$-ray signal, detected with Konus-Wind in an offline analysis. Based on its X-ray-to- $\gamma$-ray fluence ratio (see Sect. 3), GRB 020410 lies in the soft tail of genuine GRBs and marginally qualifies as an "X-ray rich" GRB (see Heise et al. 2001). Upon detection of the GRB we started an X-ray and optical search and monitoring campaign of its afterglow. We present here the results of our study of the prompt and afterglow emission.

\section{Observations}

The BeppoSAX Wide Field Camera unit 2 (Jager et al. 1997) detected a transient event on April 10, 2002, 10:39:40 UT $\left(T_{0}\right)$ at coordinates RA $=22^{\mathrm{h}} 07^{\mathrm{m}} 04^{\mathrm{s}}$, Dec $=-83^{\circ} 49^{\prime} 18^{\prime \prime}$. The off-axis angle was $10^{\circ} 9$ in a field of view of $40^{\circ} \times$ $40^{\circ}$. Because of battery efficiency degradation the BeppoSAX Gamma-Ray Burst Monitor (GRBM) was switched off, so the association of the WFC event with a GRB could not be immediately verified. After the WFC detection, lacking a GRBM measurement we searched the ratemeters of other $\gamma$-ray monitors. The most sensitive instrument apart from the GRBM, with low energy coverage, is Konus on the Wind satellite (Aptekar et al. 1995). An off-line analysis of Konus data allowed us to confirm the GRB nature of the event (see Sect. 1.3). In order to exclude the possible recurrent/flaring nature of the source detected by the WFC, we performed a detailed archival analysis of all the BeppoSAX-WFC data around the GRB 020410 position (covering the period 1996-2001). There were 186 observing periods with a net exposure time of $2.8 \mathrm{Ms}$ ( 32 days). The $2-10 \mathrm{keV}$ flux limit was $0.2 \mathrm{mCrab}$ or $\simeq 4 \times 10^{-12} \mathrm{erg} \mathrm{cm}^{-2} \mathrm{~s}^{-1}$ (this flux level is similar to that measured by the BeppoSAX ToO observations; see below). No stable or flaring source was detected at the position of the candidate GRB. The $2^{\prime}$-radius GRB error circle determined by the WFC was disseminated $4 \mathrm{~h}$ after the event (Gandolfi 2002). This prompted a fast pointing at the error box using the BeppoSAX Narrow Field Instruments (NFIs), aimed at the detection of the early X-ray afterglow (Sect. 2), and rapid searches for the optical (Sect. 3) and radio counterpart from the Southern hemisphere. The follow-up with the BeppoSAX-MECS (see Sect. 2) resulted in a reduction of the error region; an error box of 20" radius was disseminated on April 18. A subsequent search for a radio counterpart at $8.47 \mathrm{GHz}$ on April 23 yielded an upper limit of $200 \mu \mathrm{Jy}$ (Frail et al. 2002).

\section{Prompt emission}

\subsection{WFC data analysis}

The WFC light curves of GRB 020410 are presented in Fig. 1. The data are background subtracted using rates of 29 and $28 \mathrm{cts} \mathrm{s}^{-1}$ for the lower and higher energy bands respectively. These values were determined from the light curve portions immediately preceding the event and are appropriate for the part of the detector illuminated by the source. Note that, due to the slow rise of the GRB flux at its onset, we set the start time by interpolating the rise of the first peak with an exponential function; $T_{0}$ corresponds to the time when the

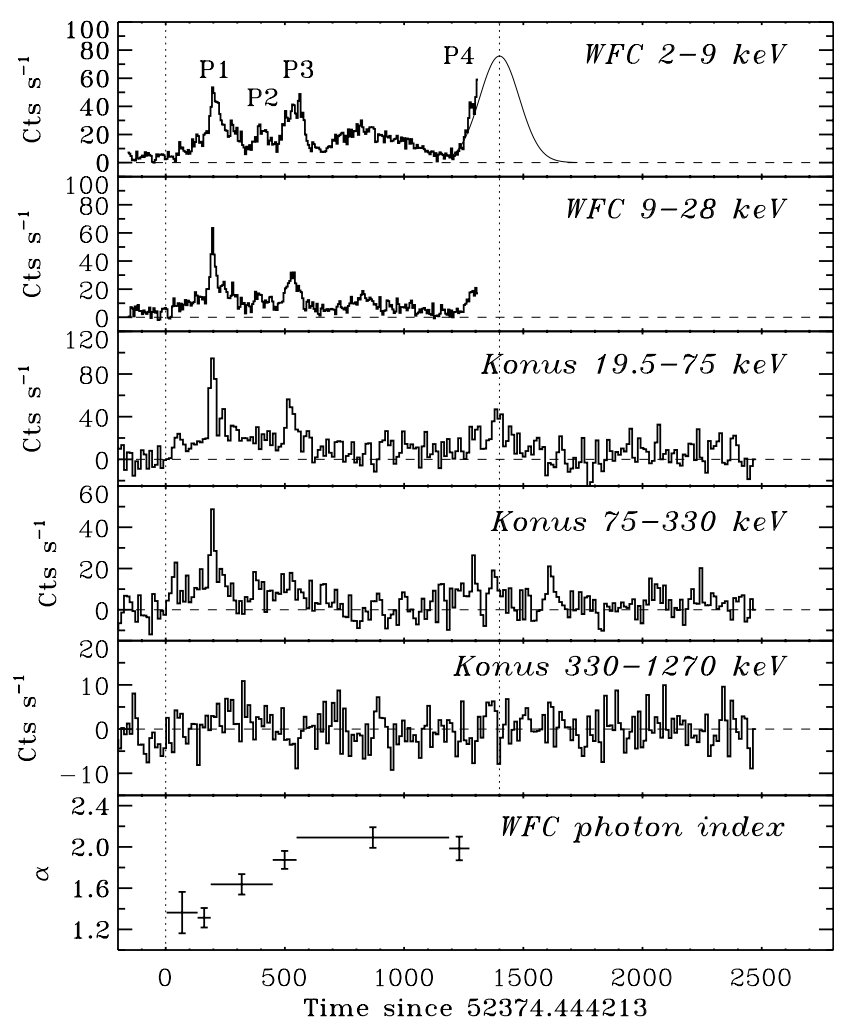

Fig. 1. Upper 5 panels: photon count rates in two WFC bands and 3 Konus bands. A time interval of $1.48 \mathrm{~s}$ was subtracted from the Konus times to account for the delay in light travel time between Wind and BeppoSAX. The extrapolated (Gaussian) last peak is shown in the $2-9 \mathrm{keV}$ light curve. The lowest panel shows the photon index resulting from a power law fit to the WFC spectra only.

function reaches the zero counts level. Although this method is somewhat arbitrary, it is reliable, because our results do not change significantly if we move forward the start time by one minute. In the $16.1 \mathrm{~h}$ time interval prior to the event, no signal was detected. The first sign of activity is distinguishable at high energies about $15 \mathrm{~s}$ before the adopted start time. The event consists of four main pulses (hereafter designated as $\mathrm{P} 1, \mathrm{P} 2, \mathrm{P} 3$ and $\mathrm{P} 4)$, the first of which, $\mathrm{P} 1$, has a maximum peak flux of $(1.9 \pm 0.4) \times 10^{-8} \mathrm{erg} \mathrm{cm}^{-2} \mathrm{~s}^{-1}$ in the 2-10 keV range. Earth occultation caused the end of the observation when the GRB was still active and rising, therefore we can only put a lower limit on its duration and fluence, $T_{\mathrm{d}} \gtrsim 1300 \mathrm{~s}$ and $4.7 \times 10^{-6} \mathrm{erg} \mathrm{cm}^{-2}(2-10 \mathrm{keV})$, respectively. The truncated peak $(\mathrm{P} 4)$ has a $2-10 \mathrm{keV}$ flux $F_{\mathrm{p}}=(2.5 \pm 0.4) \times$ $10^{-8} \mathrm{erg} \mathrm{cm}^{-2} \mathrm{~s}^{-1}$ and it is then the highest peak in this range.

However we can make reasonable assumptions to estimate the missing flux due to the truncated peak in the WFC:

- the peak shape is Gaussian, i.e. symmetric (usually the decay is slower than the rise so this gives a lower limit to the pulse width);

- it is in phase with the peak visible in the Konus G1 band (see below);

- its FWHM conforms to the law FWHM $\propto E^{\alpha}$ (see below);

- the total counts are proportional to those of P3 when compared to the Konus (G1) one, i.e. the spectral shape is the same as for P3. 
The extrapolated light curve is shown in Fig. 1. We find that, including the reconstructed portion of the light curve, the event lasts $\sim 1550 \mathrm{~s}$; the total counts are increased with respect to the measured ones by $22 \%$ in the energy band $2-9 \mathrm{keV}$ and by $21 \%$ in $9-28 \mathrm{keV}$. The estimated fluences in $2-10$ and $2-28 \mathrm{keV}$ become $(5.7 \pm 0.5) \times 10^{-6}$ and $(1.1 \pm 0.1) \times 10^{-5} \mathrm{erg} \mathrm{cm}^{-2}$ respectively.

With its large X-ray fluence, GRB 020410 ranks in the top $3.4 \%$ of the BeppoSAX-WFC GRB+XRFs sample.

The temporally resolved WFC spectra are described by simple power laws $N_{E} \propto E^{-\alpha}$ with $\alpha$ varying between $\simeq 1.4$ at the start to $\simeq 2.1$ from about $T_{0}+700 \mathrm{~s}$ onward (see Fig. 1, bottom panel). This index range is typical for GRBs (Preece et al. 2002; Frontera et al. 2000), even though on the soft side of the distribution, and its change indicates a hard-to-soft evolution. No evidence of intrinsic absorption is found in any of the spectra; the average $2 \sigma$ upper limit on the intrinsic hydrogen equivalent column density is $3.3 \times 10^{21} \mathrm{~cm}^{-2}$.

\subsection{Konus data analysis}

Since the event did not trigger Konus in a burst data acquisition mode, we analyzed the non-triggered data, pertaining to photon count rates at $2.944 \mathrm{~s}$ resolution in 3 bands: $19.5-75 \mathrm{keV}$ (G1 band), 75-330 keV (G2) and 330-1270 keV (G3). The corresponding time light curves are shown in Fig. 1. The background was estimated in an analogous way as for the WFC, giving 2929 (G1), 1017 (G2) and 418.6 (G3) cts/2.944 s. These are close to the nominal values, which have an accuracy of $2 \%$. The event is clearly detected by Konus up to a few hundred $\mathrm{keV}$. This confirms the GRB nature of the event detected by the BeppoSAX-WFC. There appears to be an initial signal in the G2 band at about the same time as in the upper WFC band, and the main Konus peak coincides with the first WFC peak. The other WFC peaks are also detected with Konus. A fortunate circumstance is that Konus continued observing after the WFC pointing ended. The Konus data in the G1 band suggest that the X-ray flux continues to increase for about $100 \mathrm{~s}$ past the end of the WFC observation. Therefore the absolute flux maximum in X-rays was most likely missed.

The spectra obtained by combining the data in the 3 Konus channels are not described by a simple power law. The flux in the G3 band must be slightly suppressed to satisfy the observed count rate. If this is modeled by a power law with an exponential cut-off, we find that a shallow e-folding cutoff energy of $900 \mathrm{keV}$, in combination with a photon index of 1.8, is sufficient to explain this suppression. WFC and Konus are not intercalibrated instruments. Fortunately they have an overlapping energy band so to obtain the flux in the Konus band we allowed for a free normalization with respect to the WFC in a joint fit (keeping constant photon index and cut-off energy). The average photon spectrum of WFC and Konus data may be described by the law $1.0 E^{-1.8} \exp (-E / 900) \mathrm{ph} \mathrm{cm}^{-2} \mathrm{~s}^{-1} \mathrm{keV}^{-1}$. The $15-1000 \mathrm{keV}$ fluence is $2.8 \times 10^{-5} \mathrm{erg} \mathrm{cm}^{-2}$ (for $1550 \mathrm{~s}$ ) and the peak flux $1.0 \times 10^{-7} \mathrm{erg} \mathrm{cm}^{-2} \mathrm{~s}^{-1}$. The $2-10 \mathrm{keV}$ to $15-1000 \mathrm{keV}$ fluence and peak flux ratios are then $\sim 0.20$ and $\sim 0.25$ respectively. The fluence in the standard BATSE energy

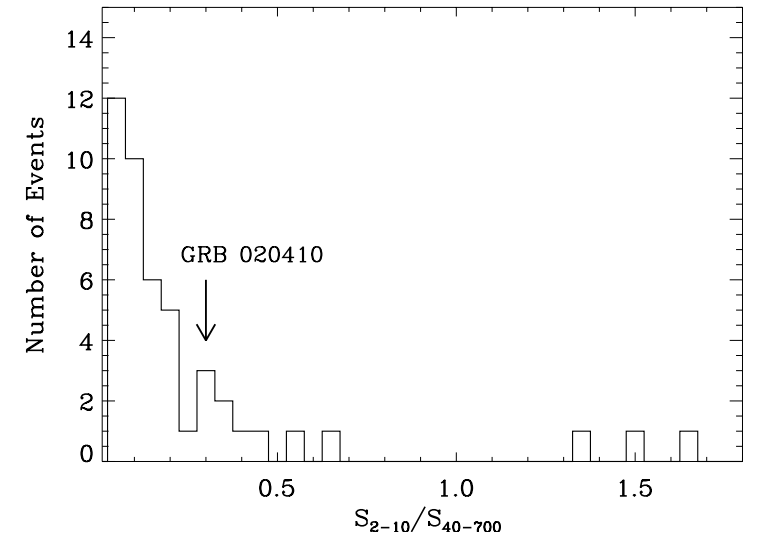

Fig. 2. Histogram of $2-10 / 40-700 \mathrm{keV}$ fluence ratio for the BeppoSAX GRBs.

range, $50-300 \mathrm{keV}$, is $\sim 1.3 \times 10^{-5} \mathrm{erg} \mathrm{cm}^{-2}$, so that in this case the soft to hard fluence ratio is $\sim 0.44$. If we consider the BeppoSAX-GRBM energy band $40-700 \mathrm{keV}$ instead, the fluence is $\sim 2.1 \times 10^{-5} \mathrm{erg} \mathrm{cm}^{-2}$, giving a ratio of $\sim 0.27$.

Though these values are not particularly high, they put GRB 020410 in between typical GRBs and X-ray rich events (see Fig. 2, Feroci et al. 2001 and Fig. 3 in Heise et al. 2001). Finally, by integrating the background-subtracted Konus light curves from $t=1550$ to $t=2500 \mathrm{~s}$ we detect a marginally significant counts excess of $\sim 1.6$ and $2.5 \sigma$ for the $\mathrm{G} 1$ and G2 bands respectively. No excess is found in G3.

\section{BeppoSAX MECS observations}

At the epoch of GRB020410, among the BeppoSAX-NFIs only the MECS was operational because of the critical status of the satellite batteries at the end of its lifetime. The error box of the event was observed with the MECS in Target-of-Opportunity (ToO) mode on 11 April 2002, 06:53:21 UT and on 12 April 2002, 16:48:44 UT, i.e. 20.2 and $54.3 \mathrm{~h}$ after the burst onset, respectively. The observations lasted 7.5 and $5.3 \mathrm{~h}$ with exposure times of $\sim 23$ and $\sim 15 \mathrm{ks}$, respectively. A third pointing was attempted but it failed because of problems in the satellite attitude control system.

A relatively bright source was detected within the WFC error circle at coordinates $\mathrm{RA}=22^{\mathrm{h}} 06^{\mathrm{m}} 25^{\mathrm{s}} .8$, Dec $=$ $-83^{\circ} 49^{\prime} 27^{\prime \prime}$ (1SAX J2206.4-8349). The source showed a clear fading between the two epochs. We therefore identify it with X-ray afterglow emission. Thanks to the new calibration of the MECS instrument (Perri \& Capalbi 2002) we were able to set a $90 \%$ confidence error circle of $20^{\prime \prime}$ radius. This position was $\simeq 1$. 3 away from the center of the WFC error circle (Gandolfi 2002).

\subsection{Spectral analysis}

By fitting the MECS spectra of both ToOs with single power laws we obtain acceptable results. The photon index is quite stable around $\alpha \simeq 2\left(N_{E}=K E^{-\alpha}\right)$, typical for GRBs. The $2-10 \mathrm{keV}$ average flux is $(3.4 \pm 0.2) \times 10^{-12}$ and $(1.5 \pm 0.1) \times$ $10^{-12} \mathrm{erg} \mathrm{cm}^{-2} \mathrm{~s}^{-1}$ for the first and second ToO, respectively. 


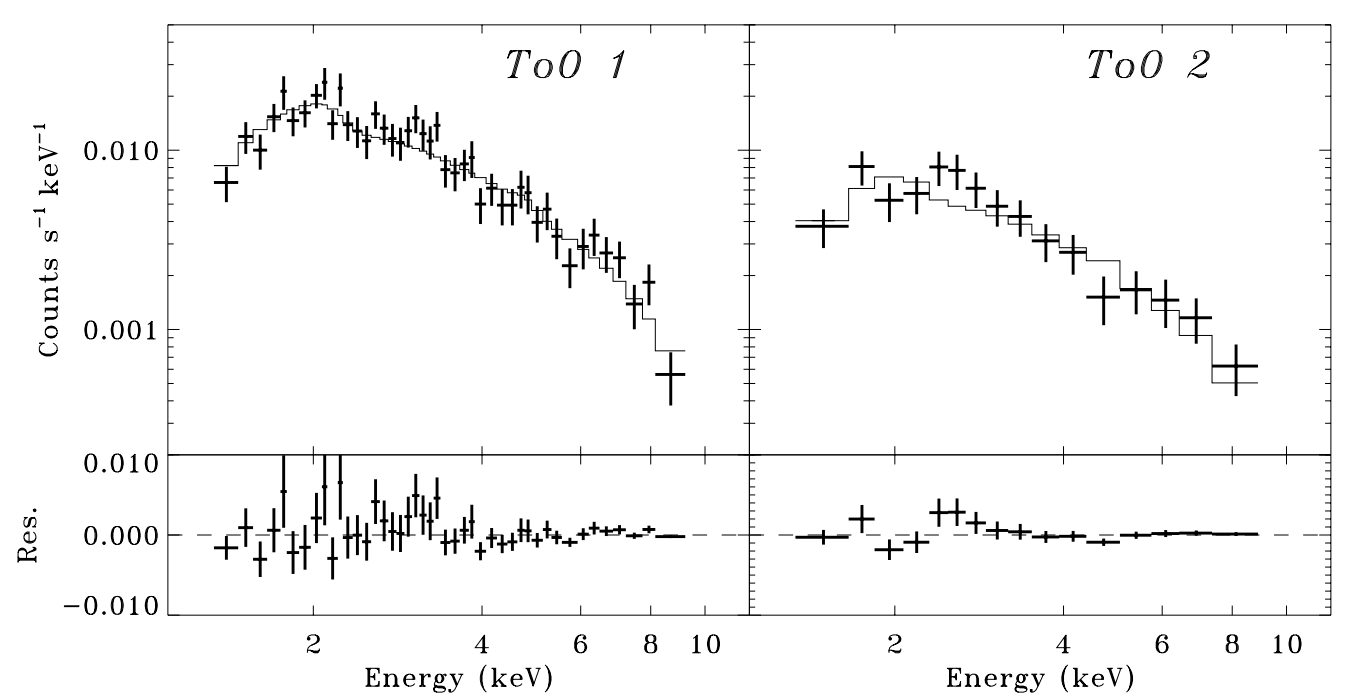

Fig. 3. Spectra for the two BeppoSAX-MECS ToOs. The data are fitted with a power law plus a Galactic $N_{\mathrm{H}}$. Note the excess around $3 \mathrm{keV}$ in both ToOs.

Because of the lack of the LECS data we included in our analysis the lower energy MECS data down to $1.3 \mathrm{keV}$ (instead of the usual $1.6 \mathrm{keV}$ ). We verified the validity of the response matrix and data quality in this range. No extra absorption above the Galactic one is detected. However, driven by systematic deviations in the residuals, we tried to fit additional components. In particular, we added a Gaussian component to model the bump visible around $3 \mathrm{keV}$ (see Fig. 3). In the first ToO the line position is $3.07_{-0.17}^{+0.15} \mathrm{keV}$ ( $2 \sigma$ confidence). The F-test chance probability of fit improvement is $1.7 \%(2.1 \sigma)$. In the second ToO the line position is $2.56_{-0.15}^{+0.19} \mathrm{keV}$ with a F-test chance probability of $3.5 \%(1.8 \sigma)$. Assuming the line detection is authentic, these results suggest that its energy is changing, although a blend of multiple components could mimic this behavior instead (see below). Next, we fitted the first ToO split in two, both with a simple power law and with an additional Gaussian. Also in this case the line position, though at $2 \sigma$ level, is not stable, being $2.96_{-0.28}^{+0.18} \mathrm{keV}$ in the first and $3.36_{-0.43}^{+0.20} \mathrm{keV}$ in the second half of the ToO. The F-test chance probabilities for the two sections are $5.8 \%$ and $4.6 \%$, respectively. In Table 1 a summary for all these fits is presented. The statistics are too poor to confirm the presence of emission lines, because the above reported results are only $2 \sigma$ results. We performed 3000 spectral simulations (1000 for each of the halves of ToO 1 and 1000 for ToO 2) of a power law with parameters fixed to those obtained for our data and checking the $\chi^{2}$ improvement if we added a Gaussian line of width $0.1 \mathrm{keV}$ falling in either the whole MECS bandpass $2-9 \mathrm{keV}$ (where we detect signal) or 2.2-4 keV (line position). For the latter range we derive chance probabilities not significantly different from those derived from the F-test on the observed data. For the wider energy range the statistical significance is a factor 1.5 lower. By randomly constructing sets consisting of one simulated spectrum of the first half of ToO 1 , one of the second half of ToO 1 and one of ToO 2, we computed the percentage of sets in which: 1. a line is detected in all three spectra with line energy centroids within $\sim 1.2 \mathrm{keV}$ of the observed (fitted) ones; and 2. the significance is equal to or greater than the observed ones. The result is $0.2 \%$.
Table 1. Spectral analysis of GRB 020410; the $N_{\mathrm{H}}$ is fixed at the Galactic value $8 \times 10^{20} \mathrm{~cm}^{-2}$ and the line width at $0.1 \mathrm{keV}$.

\begin{tabular}{cccccc}
\hline \hline ToO & $\begin{array}{c}K_{\mathrm{PL}}^{a} \\
\times 10^{-3}\end{array}$ & $\alpha$ & $\begin{array}{c}E_{\text {line }} \\
(\mathrm{keV})\end{array}$ & $\begin{array}{c}K_{\text {line }}^{a} \\
\times 10^{-5}\end{array}$ & $\chi_{n}^{2}$ \\
\hline 1 & $1.44_{-0.20}^{+0.22}$ & $2.05_{-0.13}^{+0.12}$ & - & - & 0.93 \\
1 & $1.38_{-0.20}^{+0.22}$ & $2.05_{-0.12}^{+0.13}$ & $3.07_{-0.17}^{+0.15}$ & $2.4_{-1.4}^{+1.8}$ & 0.80 \\
$1 \mathrm{a}$ & $1.57_{-0.30}^{+0.34}$ & $2.06_{-0.16}^{+0.17}$ & - & - & 0.81 \\
$1 \mathrm{a}$ & $1.49_{-0.31}^{+0.35}$ & $2.05_{-0.18}^{+0.19}$ & $2.96_{-0.28}^{+0.18}$ & $2.8_{-2.1}^{+3.1}$ & 0.69 \\
$1 \mathrm{~b}$ & $1.27_{-0.25}^{+0.28}$ & $2.01_{-0.17}^{+0.16}$ & - & - & 0.80 \\
$1 \mathrm{~b}$ & $1.24_{-0.25}^{+0.28}$ & $2.02_{-0.18}^{+0.18}$ & $3.36_{-0.43}^{+0.20}$ & $2.2_{-1.9}^{+2.0}$ & 0.70 \\
2 & $0.53_{-0.14}^{+0.17}$ & $1.93_{-0.24}^{+0.24}$ & - & - & 1.13 \\
2 & $0.44_{-0.15}^{+0.18}$ & $1.84_{-0.28}^{+0.28}$ & $2.56_{-0.15}^{+0.19}$ & $3.4_{-2.2}^{+1.9}$ & 0.75 \\
\hline
\end{tabular}

Note: errors are $90 \%$ confidence level.

${ }^{a}$ Power law and the Gaussian normalization constants.

\subsection{Temporal analysis}

Figure 4 shows the MECS $2-10 \mathrm{keV}$ flux measurements together with the WFC light curve binned in the same 6 time intervals as used in Fig. 1 for the spectral indices. Two possible power law decay fits $F_{x} \propto t^{-\delta}$ are shown. One is the best fit model to the MECS ToO data (also shown in the inset), giving a fairly flat decay index of $\delta=0.81 \pm 0.07$ with a reduced $\chi_{n}^{2}$ of 0.86 . The other is the model obtained by adopting the $90 \%$ upper limit of the decay index (0.92).

Following De Pasquale et al. (2002), we calculated the $1.6-10 \mathrm{keV}$ flux $11 \mathrm{~h}$ after the burst using the $\delta=0.81$ decay index. The resulting value of $7.9 \times 10^{-12} \mathrm{erg} \mathrm{cm}^{-2} \mathrm{~s}^{-1}$ is the highest among all the BeppoSAX detected GRBs, the second being GRB 010222 with $7.3 \times 10^{-12} \mathrm{erg} \mathrm{cm}^{-2} \mathrm{~s}^{-1}$ (in 't Zand et al. 2001).

\section{Optical observations}

Two optical searches were attempted 6.0 and $6.28 \mathrm{~h}$ after the GRB onset by two groups using two telescopes of the 


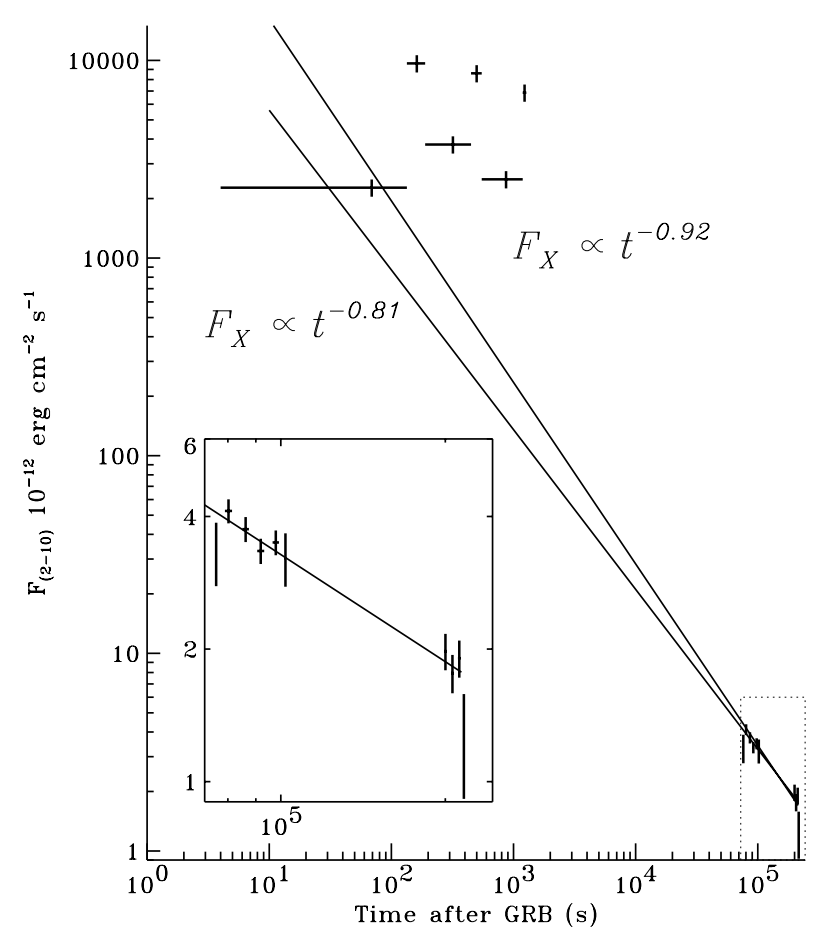

Fig. 4. X-ray (2-10 keV) WFC and MECS light curve including GRB 020410. The flatter fit is obtained using only the MECS data; the steeper one is instead constrained by using the $90 \%$ upper limit to the fitted slope.

Mount John Observatory (MJO) located in New Zealand: the MJO 0.6-m telescope and the Microlensing Observations in Astrophysics (MOA) 0.6-m telescope. Several images were collected with the two telescopes for a total of $900 \mathrm{~s}$ (unfiltered) for MJO $0.6-\mathrm{m}$ and $800 \mathrm{~s}$ (wide $B$-band filter) and $600 \mathrm{~s}$ (wide $R$-band filter) MOA $0.6-\mathrm{m}$. No positive detection was reported when compared with the POSS-II red plates down to $R \simeq 20$ (Kilmartin et al. 2002; Castro-Tirado et al. 2002). On April 17, images in the UBR bands were collected with the 3.6-m ESO telescope at La Silla (Levan et al. 2004). Further $B$ and $R$ bands observations were carried out at the 8.2-m ESO VLT on June 11 . Due to the very poor seeing conditions $\left(2^{\prime \prime}\right)$ and high airmass (1.95), a limiting $(R)$ magnitude of 24.5 for the OT is derived, which may constrain the SN maximum brightness if an underlying SN is considered. HST observations performed 27.5 and 64 days after the GRB onset revealed a variable source about $10^{\prime \prime}$ away from the MECS X-ray position (Fruchter et al. 2002; Levan et al. 2004). The decay index was $\delta \simeq 1.65$. The reported $V$ magnitudes, if converted to $R$ using the relation of Šimon et al. (2001), become 24.9 and 26.5 respectively. Assuming a constant decay index, this would imply $R \simeq 16.56 \mathrm{~h}$ after the burst. Driven by this finding, a more accurate analysis of the MOA data was performed leading to the detection of the optical transient with $R \simeq 21$ and $B>21.5$ (see Fig. 5). Re-analysis of the ESO 3.6-m $R$-band observation performed in April 17.31-17.35 (i.e. 6.87 days after the burst), also revealed a $2 \sigma$ level source at $R \approx 24$ compatible with the $R=24.3$ mag galaxy 0.5 away from the OT detected by HST (Levan et al. 2004). Then we can adopt a limit of $R=25$ for the OT in the ESO image so that the decay index between the

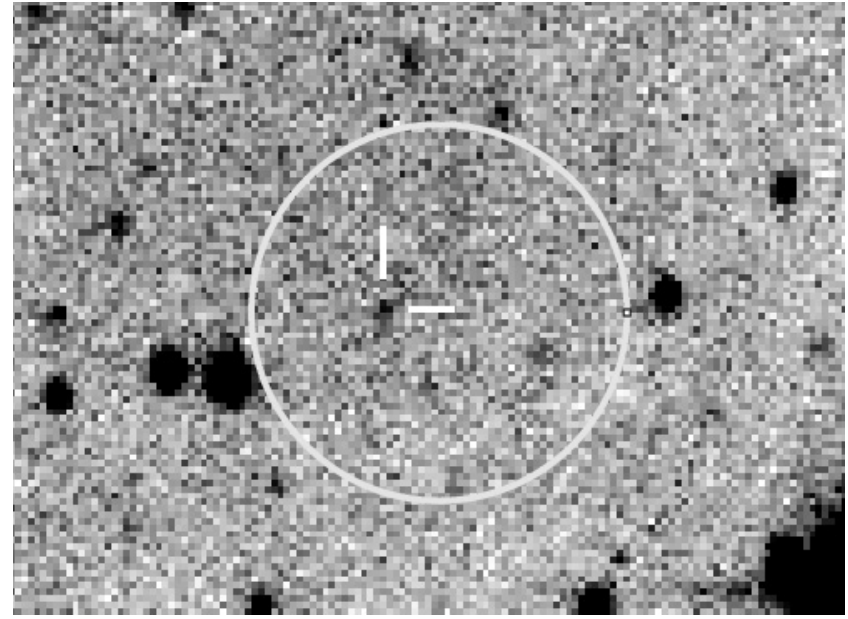

Fig. 5. $600 \mathrm{~s}$ co-added $R$-band image of the GRB 020410 field taken with the MOA 0.6-m telescope $6.25 \mathrm{~h}$ after the burst. The larger circle represents the MECS $20^{\prime \prime}$ error box; the smaller one highlights the OT at the HST position. Norh is up and East to the left.

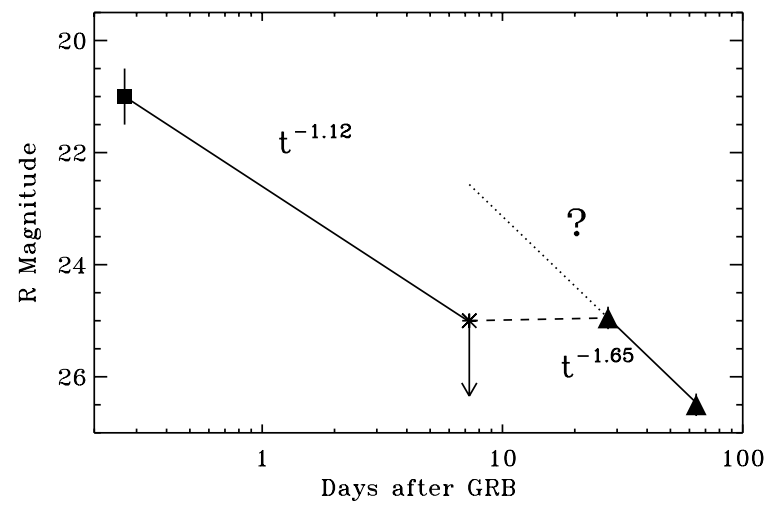

Fig. 6. $R$-band magnitudes and possible time evolution of the optical transient of GRB 020410. The first point is the MOA 0.6-m detection. The asterisk is the ESO 3.6-m (see also Levan et al. 2004) upper limit. Filled triangles are the HST measurements converted using $R-V \simeq 0.4$ (Šimon et al. 2001). The early time decay law marginally agrees with that observed in X-rays.

MJO and ESO observations is $\geq 1.1$. The optical lightcurve between 10 and 20 days must clearly deviate from this decline (see Fig. 6), and this may be accounted for if the flux increases due to the emergence of another emission component (e.g. a supernova; Woosley 1993) or a refreshed shock (Panaitescu et al. 1998). Whichever is the correct interpretation, like for GRB 991208 (Castro-Tirado et al. 2001), a double break in the optical light curve must be invoked. A detailed study of the OT behavior and possible explanations for the late time re-bump are reported in Levan et al. (2004).

\section{Discussion}

We are aware of only one GRB promptly detected in the BATSE data (and Ulysses) lasting more than $\sim 800 \mathrm{~s}$ : GRB971208 (Connaughton et al. 1997). Actually its true duration of $\sim 2000 \mathrm{~s}$ (and softness) was reported at a later time (Giblin et al. 2002). This event is also detected with 
Konus-Wind and we confirm its duration of $\sim 2500$ s. Softness and slow rise act against prompt detection of this type of events. Further investigations on the BATSE data triggered by BeppoSAX-WFC findings led to the discovery of two more cases of such long-lived GRBs (in 't Zand et al. 2003). GRB 020410 is by far the longest GRB event for which X-ray afterglow emission and an optical counterpart (though weak) have been discovered. The long duration of GRB 020410 can be considered "tail of the distribution" rather than a peculiar case, though peculiar circumstances are required. Like the other very long GRBs detected by the WFC (in 't Zand et al. 2003), this event shows a high ratio between X-ray and $\gamma$-ray fluence, although it cannot be classified as an X-ray rich GRB. In addition, apart from the high X-ray content, $\mathrm{X}$-ray rich GRBs are also characterized by the lack of optical afterglow (not true for the X-ray flash 030723 and possibly 020903), which is not the case for GRB 020410. We note, however, that X-ray richness and lack of optical detection might not be linked as the rapidly fading OT of the "not-quite" X-ray rich GRB 021211 has demonstrated (Crew et al. 2003). Spectroscopically, GRB 020410 does not show peculiarities.

It is interesting to consider if, and to which extent, the external shock component contributes to the prompt emission and whether it can be responsible for its exceptionally long duration.

Unlike what is observed for several GRBs (e.g. Costa 1999) the backward extrapolation of the afterglow fading law of GRB 020410 is inconsistent with the flux measured during the last part of the prompt emission (see Fig. 4). This may be linked to the extremely long duration of the event and prevents us from deriving an indication of the afterglow emission onset time. We can estimate an upper limit to the $2-10 \mathrm{keV}$ fluence of the afterglow if, following Frontera et al. (2000), we assume that the afterglow emission starts at $63 \%$ of the duration of the GRB and thus we integrate the fading law between $973 \mathrm{~s}$ and $1 \times$ $10^{6} \mathrm{~s}$. The result is $1.96 \times 10^{-6} \mathrm{erg} \mathrm{cm}^{-2}$, corresponding to about $34 \%$ of the fluence measured in the prompt event in the same energy range and to about $9 \%$ of the prompt fluence in 40-700 keV. These values are well within the observed range of normal GRBs (Frontera et al. 2000), even though the peculiar nature of this event does not allow us to confidently adopt average values in its description.

Alternatively, it is possible to identify the onset of the external shock at $t \sim 500 \mathrm{~s}$ when the spectrum of the prompt emission becomes consistent with the late time MECS spectra. In the simple case in which the fireball is homogeneous and thin, the GRB variability should be suppressed and the lightcurve be described as a power law initially rising as $t^{2}$ and then smoothly turning over to a decay slope which depends on the spectral range and dynamics of the fireball (Sari \& Piran 1999). In fact the lightcurve of GRB 020410 is highly variable after the spectral transition, showing a prominent emission episode at $t \sim 1500 \mathrm{~s}$ (P4 in Fig. 1). This behavior can be understood if the inner engine does not turn off at the end of the gamma-ray phase, but releases a sizable amount of energy at $t \sim 1500 \mathrm{~s}$. This late emission, however, should be inefficient in the production of $\gamma$-rays or, in terms of the internal-external shock scenario, it should avoid the internal shock phase. The time $t \sim 1500 \mathrm{~s}$ is not the deceleration time of the fireball, but the delay with which the inner engine released the fireball component that re-energized the external shock to produce the $\mathrm{P} 4$ rebrightening. The cause of the lack of $\gamma$-ray emission associated with the delayed energy release is not clear and, lacking WFC data for the P4 episode, it is difficult to constrain observationally; though the slight count excess in the Konus soft band could be a hint. The delayed energy release may however be associated to the recycling of the energy wasted while the relativistic jet propagates into the host star (Mészáros \& Rees 2001; Ramirez-Ruiz et al. 2002). In that case, the acceleration of the delayed fireball takes place at the surface of the star, and is therefore characterized by a variability timescale many orders of magnitude larger than that of the jet, effectively preventing the occurrence of internal shocks.

We are therefore left with one strong conclusion and several possibilities. The long duration of the prompt emission must be due to a long lasting activity of the inner engine. Even if the external shock is assumed to contribute sizably to the emission at $t \gtrsim 500 \mathrm{~s}$, the variability of the lightcurve can be explained only if the shock is continuously refreshed by fresh injections of energy. However, whether the emission we see comes from internal or external shocks it is not possible to tell, mainly as a consequence of the lack of BeppoSAX data at late times. The most likely situation is that, at time $t \gtrsim 500 \mathrm{~s}$, the emission we see results from the superposition of the two components. Such a long duration of the engine activity is difficult to account for in GRB progenitor models, and it points to extreme properties of the progenitor, such as a large stellar radius or a fast rotation, which both contribute to increasing the timescale of the accretion onto the compact central object.

We also note that among the other GRBs afterglows for which the extrapolation of the decay law to the prompt emission is inconsistent with the observed flux, GRB 990704 (the $\mathrm{X}$-ray richest event observed by BeppoSAX) is the only analogous case. The afterglow X-ray flux decay of XRF 031203 also shows an extrapolation below the "probable" prompt flux (Watson et al. 2004). GRB 990510, 010222, 010214 show an extrapolation above the prompt emission, which is explained by a break a few hours after the onset (Pian et al. 2001; in 't Zand et al. 2001; Guidorzi et al. 2003)

The peak width dependence as a function of the energy was tested for P1 and P3 (see Fig. 1). To this aim we produced rebinned light curves with bin size between 1 and $8 \mathrm{~s}$. Their FWHM were obtained using Gaussian fits; a $20 \%$ systematic error in their estimate was added. Using a law $F W H M=k E^{\alpha}$ (expected by the synchrotron model, Fenimore et al. 1995) for the two peaks we obtain $\alpha=-0.48 \pm 0.20$ for the P1 and $\alpha=-0.44 \pm 0.12$ for P3 (errors are $90 \%$ confidence level). These results are consistent with the results from the BATSE GRBs (Fenimore et al. 1995) as well as for GRB 960720 (Piro et al. 1998) and 990704 (Feroci et al. 2001).

Due to the paucity of data and the complexity of the optical light curve it is not possible to constrain the fireball and environment properties completely. From X-ray spectroscopy we infer the electron distribution slope $p=2.1 \pm 0.25$ under the assumption that X-rays are above the synchrotron cooling frequency. Due to the large uncertainty, the X-ray decay slope 
of $\delta_{\mathrm{X}}=0.81 \pm 0.07$ can be accounted for both in an ISM and wind environment. It is tantalizing to note, however, that the early time optical slope seems to be steeper than the X-ray one. This would fit in a wind environment scenario, consistent with the possible detection of a supernova bump at late time (Levan et al. 2004). In this case one would expect $\delta_{\mathrm{X}}=(3 p-2) / 4=$ $1.1 \pm 0.2$ and $\delta_{\mathrm{O}}=(3 p-1) / 4=1.3 \pm 0.2$, fully consistent with the X-ray slope and the optical lower limit. Even this interpretation bears some degree of uncertainty. GRB 020410 has a flux of $10.5 \mu \mathrm{Jy}$ in $R$ and $7.9 \times 10^{-12} \mathrm{erg} \mathrm{cm}^{-2} \mathrm{~s}^{-1}$ in X-rays which falls outside the distribution found by De Pasquale et al. (2002, see their Fig. 5) and would classify it as a dark GRB (Lazzati et al. 2002). Even assuming that the cooling frequency lies exactly at the edge of the BeppoSAX band, the synchrotron spectrum would over-predict optical emission by a factor $\sim 5$. There are two possible explanations for this. One possibility is that the X-ray emission is boosted by an IC component, as in the case of GRB 000926 (Harrison et al. 2001). This would require a moderately dense environment, either uniform or stratified. Alternatively, the optical emission may be extincted by a sizable amount of dust in the host galaxy, with $A_{\mathrm{V}} \sim 2$. This would correspond, for a Galactic mixture, to a column density $N_{\mathrm{H}} \sim 3 \times 10^{21} \mathrm{~cm}^{-2}$, consistent with the upper limit derived from X-ray spectroscopy. The lack of constraints on the optical spectrum prevents us from reaching a definite conclusion. The optical spectrum should be bluer in the case of IC emission and red in the case of dust obscuration.

Assuming that the emission line in the MECS spectra is real and due to fluorescence of H-like iron (rest energy of $6.97 \mathrm{keV}$ ), the change in line position can be explained by a variable iron recombination edge having its maximum in the second half of ToO 1 (or later, but before ToO 2). In fact, if we derive the redshift from the line position in ToO 2 we obtain $z \simeq 1.7$ which leads to a recombination edge of $\sim 3.4 \mathrm{keV}$. Also the ratio between the iron recombination edge rest energy, $9.28 \mathrm{keV}$, and $6.97 \mathrm{keV}$ is $\simeq 1.3$, like the ratio of the ToO $1 \mathrm{~b}$ over ToO 2 line energies. Again, our statistics does not allow us to perform a simultaneous fit for a Gaussian and a recombination edge line. However this hypothesis appears to be in agreement with the data.

As no direct $z$ measurement exists for GRB 020410, we calculated the peak energy $E_{\mathrm{p}}$ in the $v F_{v}$ spectrum and the isotropic energy $E_{\mathrm{rad}}$ for a grid of $z$ values. We then compared the results with the relation reported by Amati et al. (2002). We find that the relation is satisfied (with a discrepancy level $<20 \%$ ) for $0.9<z<1.5$ and $1.1 \times 10^{53}<E_{\mathrm{rad}}<3.0 \times 10^{53} \mathrm{erg}$. This range of $z$ would exclude the value of 0.5 obtainable by assuming a 1998bw-like SN re-bump (Levan et al. 2004) and is marginally in agreement with the value derived above. Even assuming that our flux estimate for the missing part of the X-ray light curve must be increased by an extra $20 \%$ (which is unlikely), the lower limit for $z$ becomes 0.6. Besides this, we note that $z \simeq 0.5$ together with the reported magnitude of $V \simeq 28.7$ for the host galaxy (Levan et al. 2004) would place it at the very low end of the galaxy luminosity function $\left(M_{\mathrm{V}}=-14.3\right)$, which is unusual for GRB hosts; this is independent of considering the X-ray spectrum derived $N_{\mathrm{H}} \lesssim 3 \times 10^{21} \mathrm{~cm}^{-2}$ "local" or "global".
Acknowledgements. This research was supported by the Italian Space Agency (ASI) and Consiglio Nazionale delle Ricerche (CNR). BeppoSAX was a major program of ASI with participation of the Netherlands Agency for Aerospace Programs (NIVR). All authors warmly thank the extraordinary teams of the BeppoSAX Scientific Operation Center and Operation Control Center for their enthusiastic support of the GRB program. One of of us (A.J.C.T.) is grateful to the MOA project for granting part of the observing time to the GRB follow-up program and thanks P. Kilmartin, A. Gilmore, Ph. Yock and B. Nelson for their assistance. LN thanks prof. F. Frontera for several useful discussions. This research made use of observations retrieved from the ESO data archive. The Konus-Wind experiment was supported by Russian Space Agency contract and RBRF grant \# 03-02-17517.

\section{References}

Amati, L., Frontera, F., Tavani, M., et al. 2002, A\&A, 390, 81

Aptekar, R. L., Frederiks, D. D., Golenetskii, S. V., et al. 1995, Space Sci. Rev., 71, 265

Barraud, C., Olive, J.-F., Lestrade, J. P., et al. 2003, A\&A, 400, 1021

Björnsson, G., Hjorth, J., Pedersen, K., \& Fynbo, J. P. U. 2002, ApJ, 579, L59

Castro-Tirado, A., Brandt, S., Lund, N., et al. 1994, in Gamma-ray Burst Workshop, ed. G. J. Fishman, J. J. Brainerd, \& K. Hurley, AIP, 307, 17

Castro-Tirado, A., Sokolov, V. V., Gorosabel, J., et al. 2001, A\&A, 370,398

Castro-Tirado, A., Gorosabel, J., Castro Ceron, J. M., Nelson, B., \& Tristram, P. 2002, GCN Circ., No. 1355

Connaughton, V., Kippen, R. M., Preece, R., \& Hurley, K. 1997, IAU Circ., 6785

Costa, E. 1999, A\&AS, 138, 425

Crew, G. B., Lamb, D. Q., Ricker, G. R., et al. 2003, ApJ, 599, 387

De Pasquale, M., Piro, L., Perna, R., et al. 2002, ApJ, 592, 1018

Dermer, C. D., Chang, J., \& Bottcher, M. 1999, ApJ, 513, 656

Fenimore, E. E., in 't Zand, J. J. M., Norris, J. P., Bonnell, J. T., \& Nemiroff, R. J. 1995, ApJ, 448, L101

Feroci, M., Antonelli, L. A., Soffitta, P., et al. 2001, A\&A, 378, 441

Frail, D., Wieringa, M. H., Berger, E., \& Wark, R. 2002, GCN Circ., No. 1380

Frontera, F., Amati, L., Costa, E., et al. 2000, ApJS, 127, 59

Fruchter, A., Levan, A. J., Burud, I., \& Nugent, P. E. 2002, GCN Circ., No. 1453

Fynbo, J. P. U., Sollerman, J., Hjorth, J., et al. 2004, ApJ, accepted [arXiv: astro-ph/0402240]

Gandolfi, G. 2002, GCN Circ., No. 1349

Giblin, T. W., Connaughton, V., van Paradijs, J., et al. 2002, ApJ, 570, 573

Granot, J., Panaitescu, A., Kumar, P., et al. 1999, ApJ, 570, L61

Guidorzi, C., Frontera, F., Montanari, E., et al. 2003, A\&A, 401, 491

Heise, J., in 't Zand, J. J. M., Kippen, M., \& Woods, P. 2001, in ESO Proc. Ser., Gamma-Ray Bursts in the Afterglow Era, ed. E. Costa, F. Frontera, \& J. Hjorth (Berlin Heidelberg: Springer), 16

Huang, Y. F., Dai, Z. G., \& Lu, T. 2002, MNRAS, 332, 735

Jager, R., Mels, W. A., Brinkman, A. C., et al. 1997, A\&AS, 125, 557

Harrison, F. A., Yost, S. A., Sari, R., et al. 2001, ApJ, 559, 123

Kilmartin, P., \& Gilmore, A. 2002, GCN Circ., No. 1350

Lazzati, D., Covino, S., \& Ghisellini, G. 2002, MNRAS, 330, 583

Levan, A., Nugent, P., Fruchter, A., et al. 2004, ApJ, submitted [arXiv: astro-ph/0403450]

Mészáros, P., \& Rees, M. J. 2001, ApJ, 556, L37 
Mochkovitch, R., Daigne, F., Barraud, C., \& Atteia, J.-L. 2003, in Sari, R., \& Piran, T. 1999, ApJ, 520, 641

Proc., GRBs in the afterglow era: 3rd Rome workshop (2002), ed. L. Piro, F. Frontera, N. Masetti, \& M. Feroci, in press [arXiv: astro-ph/0303289]

Nicastro, L., Piro, L., Gandolfi, G., et al. 2002, GCN Circ., No. 1374

Paciesas, W. S., Meegan, C. A., Pendleton, G. N., et al. 1999, ApJS, 122,465

Panaitescu, A., Mészáros, P., \& Rees, M. J. 1998, ApJ, 503, 314

Perri, M., \& Capalbi, M. 2002, A\&A, 396, 753

Pian, E., Soffitta, P., Alessi, A., et al. 2001, A\&A, 372, 456

Piro, L., Amati, L., Antonelli, A., et al. 1998, A\&A, 331, L41

Preece, R. D., Briggs, M. S., Mallozzi, R. S., et al. 2000, ApJS, 126, 19

Prochaska, J., Bloom, J. S., Chen, H. W., et al. 2003, GCN Circ., 2482

Ramirez-Ruiz, E., Celotti, A., \& Rees, M. J. 2002, MNRAS, 337, 1349

Šimon, V., Hudec, R., Pizzichini, G., \& Masetti, N. 2001, A\&A, 377, 450

Soderberg, A. M., Kulkarni, S. R., Berger, E., et al. 2003, ApJ, submitted [arXiv: astro-ph/0311050]

Yamazaki, R., Yoka, K. S., \& Nakamura, T. 2002, ApJ, 571, L31

Watson, D., Hjorth, J., Levan, A., et al. 2004, ApJ, accepted [arXiv: astro-ph/0401225]

Woosley, S. E. 1993, ApJ, 405, 273

in 't Zand, J. J. M., Kuiper, L., Amati, L., et al. 2001, ApJ, 559, 710

in't Zand, J. J. M., Heise, J., Kippen, R. M., et al. 2003, in Proc., GRBs in the afterglow era: 3rd Rome workshop (2002), ed. L. Piro, F. Frontera, N. Masetti, \& M. Feroci, in press [arXiv: astro-ph/0305361] 